\title{
S3_Supporting information
}

Experimental measurements of cell size $\left(W_{i t}\right)$ for each alcoholic fermentation $i$ at time $t$ were assumed to follow a linear model: $W_{i t}=H+w . t+\varepsilon_{i t},\left[\varepsilon_{i t}\right]$ i.i.d. $\sim \mathrm{N}\left(0, \sigma_{w, i}{ }^{2}\right)$ between $t . N_{\max }$ and the time at which $93 \%$ of the total amount of $\mathrm{CO}_{2}$ was released. All the data points outside this time range were discarded for the following reasons: (i) cell size tended to increase before $t . N_{\max }$ but we did not have enough experimental data points before $t N_{\max }$ to fit such a model; (i) cells tended to flocculate at the end of the fermentation and we observed that the data were no more reliable beyond about $93 \%$ of total amount of $\mathrm{CO}_{2}$ released. Again, after fitting the model, the homogeneity and independence of the residuals was checked by pooling all fermentations and plotting the residuals against the fitted values. 